\title{
The psychological effect of the COVID-19 pandemic on rural appalachian nurses
}

\author{
Annette Lynn Ferguson, Jessica Maynard \\ Marshall University, Huntington, United States
}

Received: September 29, 2021

DOI: $10.5430 /$ jnep.v12n4p38
Accepted: November 23, $2021 \quad$ Online Published: November 29, 2021

URL: https://doi.org/10.5430/jnep.v12n4p38

\begin{abstract}
The coronavirus disease 2019 (COVID-19) pandemic has affected all individuals around the globe, spreading rapidly in late 2019. Confirmed cases of COVID-19 continue to be on the rise causing healthcare facilities to be overwhelmed. In the Appalachian region of the US, the pandemic has been devastating for the community. Healthcare workers on the frontline have had to respond to the pandemic by working directly with patients infected in both the hospital and community setting and have placed themselves and their families at risk of physical and psychological harm. Nurses are especially at risk due to their close personal contact with patients with COVID-19. The aim of this study was to assess the psychological impact of the pandemic on rural Appalachian nurses working directly with patients with COVID-19 in the acute care hospital setting. A cross-sectional design was utilized for this study. The Depression, Anxiety, Stress Scale (DASS-21) was administered to 77 nurses. The results of the study found that $66.3 \%$ of the nurses self-reported their level of depression between a moderate to extremely severe level when caring for COVID-19 patients. In addition, $72.8 \%$ of participants reported their anxiety level between moderate to extremely severe and finally, $58.5 \%$ reported their stress levels between moderate to extremely severe during this time period. Based on the results of this study, it is imperative that healthcare facilities develop strategies and interventions to address the physical and mental health needs of nurses caring for patients in these stressful work environments.
\end{abstract}

Key Words: COVID-19, Depression, Anxiety, Stress, Appalachian, Nurses

\section{INTRODUCTION}

During late 2019, coronavirus disease (COVID-19) spread rapidly across the world affecting many individuals. COVID19 is an infectious disease caused by the severe acute respiratory syndrome coronavirus 2 (SARS-CoV-2). According to the Centers for Disease Control and Prevention (CDC), this disease can lead to either a mild or severe illness with symptoms fluctuating from patient to patient. Most individuals who experience the disease will return to their previous health within weeks of recovering from COVID-19. Some experience post-coronavirus conditions such as shortness of breath, fatigue, difficulty thinking or concentrating, coughing or headaches to name a few. ${ }^{[1]}$ Worldwide, there have been over 253 million confirmed COVID-19 cases with over 5.1 million deaths reported as of November 2021. ${ }^{[2]}$ The United States (US) continues to be the county with the highest number of confirmed cases at 47 million with over 761,000 deaths as of November 2021. ${ }^{[1]}$ These numbers have continued to climb as COVID-19 remains a threat worldwide.

In the Appalachian region of the US, the pandemic has been devastating to the community. The Appalachian region is in the Eastern US and stretches from New York to Alabama. There are thirteen states that make up this area, including parts of New York, Pennsylvania, Virginia, Ohio, Kentucky, Georgia, Tennessee, Maryland, North Carolina, South Car-

*Correspondence: Annette Lynn Ferguson; Email: rn_nettie@yahoo.com; Address: Marshall University, Huntington, United States. 
olina, Alabama, Mississippi, and all of West Virginia. According to the Appalachian Regional Commission, there are over 25 million residents that live in the Appalachian region with a per capita average income of $\$ 41,155$ (national average $\$ 51,640$ ) with $15.2 \%$ living below the poverty level. Of the 25 million people that live in the Appalachian region, 2.5 million reside in rural communities. ${ }^{[3]}$ The target population for this study was nurses that work in the Appalachian region of West Virginia and Kentucky. According to the West Virginia Department of Health and Human Resources, there have been over 285,000 total confirmed cases and 4,698 deaths from COVID-19 in West Virginia as of November 2021. ${ }^{[4]}$ In Kentucky, there have been over 746,000 confirmed cases and 10,318 deaths during the same time period. ${ }^{[5]}$

Healthcare workers, especially nurses, have had to respond to the pandemic by working directly with patients infected with COVID-19 in the hospital and community settings. Frequently, critically ill patients with COVID-19 required mechanical ventilation and admission into intensive care units (ICU). With the mandatory hospital restrictions, many patients were unable to have family members at their bedside during this time. Therefore, nurses not only served as the primary caregiver for the patients but also served as the communicator between the patient and family and had to attend to the emotional needs of both. Nurses during the pandemic have had to witness considerable amounts of suffering, grief, sorrow, and death, putting them at increased risk of emotional distress.

\subsection{Significance of the problem world-wide}

In the last year, several studies have examined the impact of the COVID-19 pandemic on the mental health of healthcare workers throughout the world. A systemic review and metaanalysis were conducted on the prevalence of depression, anxiety, and insomnia among healthcare workers in China during the COVID-19 pandemic. In twelve studies reviewed, anxiety was assessed with a pooled prevalence of $23.2 \%$ and depression was assessed in 10 studies with a pooled prevalence rate of $22.8 \%$. Five studies estimated insomnia at $38.9 \%$. Results of this review found that female healthcare providers and nurses had higher rates of affective symptoms compared to male and medical staff, respectively. ${ }^{[6]}$

A study in Egypt and Saudi Arabia evaluated healthcare workers (physicians, nurses, and other healthcare workers) level of depression, anxiety, and stress during the pandemic. The study found $69 \%$ had depression, $58.9 \%$ had anxiety, and $55.9 \%$ had stress. This study also found that $37.3 \%$ had inadequate sleep (less than 6 hours a day). Results from this study observed that females working nightshift who watched

Published by Sciedu Press or read about COVID-19 more than 2 hours a day, and did not receive emotional support from family, society, and the hospital were more likely to have depression, anxiety, stress, and inadequate sleep. ${ }^{[7]}$

A large study examined the mental health among medical and nonmedical professionals during the COVID-19 pandemic in eight European countries. Across all surveyed countries, participants reported a moderate level of depression at $18 \%$ and a severe/extremely severe level of depression at $17 \%$. Regarding anxiety, $15 \%$ reported a moderate level and $22 \%$ reported a severe/extremely severe level of anxiety. In terms of stress, $14 \%$ reported moderate levels and $27 \%$ reported severe/extremely severe stress. It was found that participants in France and the United Kingdom reported higher levels in all three domains compared to participants in Germany, Austria, Switzerland, Italy, Spain, and Portugal. In this study, the most reported reasons for stress were "Uncertainty about when the epidemic would be under control" and "Worrying about inflicting COVID-19 on family" ${ }^{[8]}$

A study in Nepal assessed the level of stress, anxiety, and depression in health care workers during the outbreak. The symptoms of stress, anxiety, and depression among the participants were found to be $28.9 \%, 35.6 \%$, and $17.0 \%$, respectively. The study found females were two times more likely to have anxiety and depression than males. Nurses were two times more likely to have anxiety than doctors. Health care workers who lacked or had insufficient personal protective equipment (PPE) were almost three times more likely to have depression and those working in high-risk areas were almost two times more likely to have depression. ${ }^{[9]}$

A study in Belgium assessed the differences in mental health in physicians and nurses working in COVID and non-COVID units. Of the 647 participants of the study, $28.8 \%$ had moderate to extremely severe symptoms of depression, $41.8 \%$ had moderate to extremely severe symptoms of anxiety, and $25.1 \%$ had moderate to extremely severe symptoms of stress. In this study, nurses and females reported anxiety more frequently than physicians and male participants. In addition, nurses who were younger, had a heavy workload, and lacked social support had more symptoms of depression, anxiety, and stress. ${ }^{[10]}$

A large study conducted in Australia determined the level of depression, anxiety, and stress on critical care healthcare workers. Of the 3,770 healthcare workers who participated in the study, $21.6 \%$ reported moderate to extremely severe depression, $28.6 \%$ reported moderate to extremely severe anxiety, and $28 \%$ reported moderate to extremely severe stress scores. The study found many clinical concerns that were associated with higher scores in all three domains included not 
being clinically prepared, having inadequate workers, having to triage patients, fear of transmitting virus to friends and family, contracting the COVID-19 virus, being responsible for other staff members, or being asked to work in an area outside of expertise. [11]

A study conducted in the Philippines examined the effects of the pandemic on nurses. This study included nurses from 75 different units from a total of twenty different facilities. Greater than $90 \%$ of nurses identified they were not prepared to manage COVID-19 patients. The results from the COVID-19 Anxiety Scale found that $37.8 \%$ of nurses had dysfunctional levels of anxiety with symptoms including tonic immobility, dizziness, appetite loss, abdominal distress, and sleep disturbances. The results of this study found that nurses were impacted mentally as well as physically when caring for COVID-19 patients. ${ }^{[12]}$

\subsection{Significance of problem in United States}

The National Nurses Union conducted a survey on more than 9,200 registered nurses who worked in the United States. Overall, the participants reported having more mental health and emotional issues since the pandemic. The results of the survey found that $61 \%$ reported feeling more stressed, $57 \%$ reported an increase in anxiety, and over half felt more sad or depressed. Nurses reported they faced issues ranging from unsafe staffing levels to lack of basic infection control and prevention measures including having to reusing PPE. In addition, nurses were concerned about being exposed to coronavirus and infecting family members coupled with the delay in contact from their employers when they may have been exposed to a COVID-19 positive patient. All these issues were reported by the nurses in the survey as reasons for increased anxiety and stress during the pandemic. ${ }^{[13]}$

A study conducted in the United States, found that nursing staff in a large, California Medical Center during the COVID-19 pandemic had increased levels of depression and anxiety. Over $79 \%$ of the nurses reported feeling anxious and $77.9 \%$ reported feeling depressed during this time. This study noted that female nurses were two times more likely to be distressed compared to male nurses. Also, the study found that student nurses, licensed practical nurses, and clinical assistants had higher levels of depression and anxiety than registered nurses and nurse practitioners. ${ }^{[14]}$

A qualitative study conducted on nurses in Michigan identified themes related to stress experienced during the pandemic. The first theme identified in the study was that $29 \%$ of the participants were fearful of being exposed to COVID-19 and becoming ill, as well as passing the virus onto others, such family members, or patients. The next theme identified was related to illness/death at $38.9 \%$. This included nurses having to care for rapidly deteriorating patients, witnessing patients dying, and caring for ventilated or extremely ill patients. The next theme was work-related issues such as relationships, administration failures, and lack of supplies and training which was identified by $51.2 \%$ of the participants. Additional themes identified were stressors related to lack of PPE and supplies and dealing with the consistent unknowns during the pandemic. ${ }^{[15]}$

\subsection{Aim of study}

Several research studies identified in the literature review provided evidence that healthcare workers throughout the world and the US have been impacted by the COVID-19 pandemic. Yet, the researchers could not find any studies that focused on the impact of the pandemic on nurses working in the Appalachian region. Therefore, the aim of this study was to assess the psychological impact of the pandemic on rural Appalachian nurses working directly with patients having COVID-19 in the acute care hospital setting.

\section{Methods}

\subsection{Study design and sample}

A cross-sectional design was utilized for this study. The study was conducted from March 12, 2021, to May 17, 2021. A convenience sampling method was utilized to select participants. A total of 84 nurses completed the survey from two hospitals in the Appalachian region. Seven surveys were found to have incomplete data; therefore, they were removed from the study leaving a total of 77 participants. The response rate was estimated at $30 \%$ based on the number of eligible participants. The survey included general demographic characteristics (age, educational level, and years of experience as a nurse), work-related information (hospital, unit, and shift), and the DASS-21 instrument to measure depression, anxiety, and stress.

\subsection{Ethical consideration}

The study was approved by the institutional review board (IRB) at a mid-size University in Eastern US. Participants were invited to complete the survey via flyer and/or email on a voluntary basis. Prior to starting the survey, each participant was provided with information that explained the purpose, potential benefits/risks, and procedure for the study. Once participants consented to the study, they were provided with the survey questions. At any time, the participants were able to withdraw from the study by simply not completing the survey questions. No identifiable data was collected on participants and the survey results were keep in a secure location with the researchers being the only individuals to have access to the results. 


\subsection{Procedure}

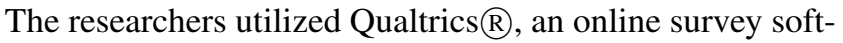
ware tool, to upload the survey questions and create a survey link with a Quick Response $(\mathrm{QR})$ code. The researchers contacted several nurse administrators at local rural hospitals and requested their participation in the study. Two hospitals agreed to participate in the study. The researchers send the administrators the $\mathrm{QR}$ code for the survey link to distribute to 282 participants that met the study criteria. The study criteria included any nurse who worked directly with COVID-19 patients prior to and during the study period. The administrators posted flyers on the nursing units and sent emails with the QR code to all participants who met the study criteria. Participation in the study was voluntary and the identity of the participants remained anonymous. The QR code for the survey remained active for three months. A reminder was sent to the nurses via email two weeks prior to the close of the survey.

\subsection{Instrument to measurement depression, anxiety, and stress}

The DASS-21 is a self-reported 21-item survey was used to measure depression, anxiety, and stress. Each of the three DASS subscales (depression, anxiety, and stress) contained 7 -item to measure these concepts. Within the depression subscale, the concepts of dysphoria, hopelessness, no self-worth, self-deprecation, lack of interest, inability to change, and lack of pleasure were assessed. Within the anxiety subscale the concepts of autonomic arousal (sweating, trembling), skeletal muscle activity, situational anxiety, and subjective experience of anxious (panic, scared) affect were assessed. Within the stress subscale the concepts of inability to relax, nervousness, being easily upset/agitated, irritable/over-reactive, and impatient were assessed. Scores for each subscale for depression, anxiety, and stress were then calculated by summing the scores for each subscale and then multiplying by two. ${ }^{[16]}$ Based on the scores derived from each subscale on the survey, the participants were divided into five categories: normal (lacking depression, anxiety, or stress), mild, moderate, severe, or extremely severe. Depression levels were categorized based on the following scores: less than 9 normal, 10-13 mild, 14-20 moderate, 21-27 severe, and 28 or higher extremely severe. Anxiety levels were categorized based on following scores: less than 7 normal, 8-9 mild, 10-14 moderate, $15-19$ severe, and 20 or higher extremely severe. Stress levels were categorized based on the following scores: less than 14 normal, 15-18 mild, 19-25 moderate, 26-33 severe, and 34 or greater extremely severe. ${ }^{[16]}$

Several researchers have utilized the DASS-21 instrument to measure depression, anxiety, and stress in various population

Published by Sciedu Press with high reliability. A study that utilized the DASS-21 instrument on hospital clinical staff had a Cronbach's alpha of $0.901,0.754$, and 0.886 for the depression, anxiety, and stress subscales. ${ }^{[17]}$ A study that utilized the DASS-21 survey on medical students had a Cronbach's alpha value of $0.81,0.89$ and 0.78 for the subscales of depressive, anxiety and stress respectively. ${ }^{[18]}$ Finally, a study that utilized the DASS-21 survey on critical care workers had a Cronbach's alpha for depression, anxiety, and stress subscales at $0.88,0.89$, and 0.90 , respectively. ${ }^{[18]}$

\subsection{Statistical analysis}

Descriptive statistics were calculated for the demographic characteristics, work-related information, and the DASS-21 survey scores. The nonparametric statistical tests, MannWhitney U and Kruskal-Wallis H, were used to evaluate the level of depression, anxiety, and stress scores among the work-related and the demographic variables, respectively. Data analysis was performed using IBM SPSS $₫$ Statistics for Windows (Version 26) and a two-sided $p$ value $<.05$ was considered statistically significant.

\section{Results}

\subsection{Participant demographic characteristics}

Almost half of participants were between the ages of 32-37 (26\%) and 26-31 (20.8\%). Fifty-seven percent of nurses had a Bachelor of Science in Nursing (BSN) degree with approximately $30 \%$ had an Associate Degree in Nursing (ADN) degree. Approximately, $9 \%$ of the nurses had a Master of Science in Nursing (MSN) degree or higher. Nurses with eight or more years of experience made up $48 \%$ of the participants with only $14 \%$ having less than one year of experience as a nurse (see Table 1).

\subsection{Work-related information}

The majority $(80.5 \%)$ of participants worked in the intensive care unit (ICU) with the other participants working on medical-surgical units caring for patients with COVID-19. Participants completing the survey predominately worked day shift at $63.6 \%$ with approximately $1 \%$ working rotating shifts. Surveys were completed by nurses from two different rural Appalachian hospitals (see Table 2).

\subsection{Psychological characteristics}

Results from the DASS-21 survey revealed that nurses selfreported various levels of depression ranging from extremely severe at $20.8 \%$, severe at $20.8 \%$, moderate at $24.7 \%$ and mild at $10.4 \%$. Various levels of anxiety were reported from $36.4 \%$ extremely severe, $14.3 \%$ severe, $22.1 \%$ moderate, and $5.2 \%$ mild. Levels of stress range from $13 \%$ extremely severe, $20.8 \%$ severe, $26 \%$ moderate, and $13 \%$ mild (see Table 
$3)$.

Table 1. Participant description

\begin{tabular}{lll}
\hline Characteristic & Number (N) & Percentage (\%) \\
\hline Age Group & 14 & 18.0 \\
$20-25$ & 16 & 20.8 \\
$26-31$ & 20 & 26.0 \\
$32-37$ & 7 & 9.1 \\
$38-43$ & 14 & 18.2 \\
$44-49$ & 4 & 5.2 \\
$50-55$ & 1 & 1.3 \\
$56-61$ & 1 & 1.3 \\
$62+$ & 77 & 100.0 \\
Total & & \\
Level of Education & 3 & 3.9 \\
LPN & 23 & 29.9 \\
ADN & 44 & 27.1 \\
BSN & 7 & 9.1 \\
Other & 77 & 100.0 \\
Total & & \\
Years of experience as nurse & & 14.3 \\
Less than 1 year & 11 & 20.8 \\
1 to 3 years & 16 & 16.9 \\
4 to 7 years & 13 & 16.9 \\
8 to 12 years & 13 & 31.2 \\
13 years or more & 24 & 100.0 \\
Total & 77 & \\
\hline Note. $L P N=$ Licensed Practical Nurse, $A D N=$ Associate Degree Nursing, \\
BSN = Bachelor of Science in Nursing.
\end{tabular}

Table 2. Work-related information

\begin{tabular}{lll}
\hline Characteristics & Number (N) & Percentage (\%) \\
\hline Hospital Employed & & \\
Hospital \#1 & 57 & 74.0 \\
Hospital \#2 & 20 & 26.0 \\
Total & 77 & 100.0 \\
Nursing Unit & & \\
Critical Care Unit (ICU) & 62 & 80.5 \\
Medical-Surgical Unit & 15 & 19.5 \\
Total & 77 & 100.0 \\
Shift Primarily Worked & & \\
Days & 49 & 63.6 \\
Nights & 27 & 35.1 \\
Rotating (Both shifts) & 1 & 1.3 \\
Total & 77 & 100.0 \\
\hline
\end{tabular}

\subsection{Comparison of variables}

The Mann-Whitney U test was calculated to compare workrelated variables (hospital, unit, shift) had on depression, anxiety, and stress scores. The Kruskal-Wallis H test was calculated to compare the impact of age, level of education, and years of experience had on depression, anxiety, and stress scores. The Mann-Whitney U test revealed no significant differences in the work-related variables and the level of depression, anxiety, or stress. The Kruskal-Wallis $\mathrm{H}$ test revealed a statistically significant difference in depression based on level of education ( Gp1, $n=3$ : LPN, Gp2, $n=23$ : ADN, Gp3, $n=44:$ BSN, Gp4, $n=7$ : other [MSN]), $\chi^{2}(3$, $\mathrm{n}=77)=10.47, p=.015$. Nurses with a higher level of education (MSN or higher) recorded lower levels of depression compared to nurses prepared at the ADN and BSN levels. The test also revealed a statistically significant difference in anxiety based on the level of education $(\mathrm{Gp} 1, \mathrm{n}=3$ : LPN, $\mathrm{Gp} 2, \mathrm{n}=23: \mathrm{ADN}, \mathrm{Gp} 3, \mathrm{n}=44: \mathrm{BSN}, \mathrm{Gp} 4, \mathrm{n}=7$ : other $[\mathrm{MSN}]), \chi^{2}(3, \mathrm{n}=77)=8.85, p=.031$. Nurses with a higher level of education (MSN or higher) recorded lower levels of anxiety compared to nurses prepared at the LPN, ADN, and BSN levels. The effect size using Cohen criteria ranged from 0.36 to 0.68 for all groups compared, indicating a medium to large effect. No other variable measured was found to be statistically significant.

Table 3. DASS-21 results

\begin{tabular}{lll}
\hline Characteristic & Number $\mathbf{( N )}$ & Percentage (\%) \\
\hline Depression & 18 & 23.4 \\
Normal & 8 & 10.4 \\
Mild & 19 & 24.7 \\
Moderate & 16 & 20.8 \\
Severe & 16 & 20.8 \\
Extremely severe & 77 & 100.0 \\
Total & & \\
Anxiety & 17 & 22.1 \\
Normal & 4 & 5.2 \\
Mild & 17 & 22.1 \\
Moderate & 11 & 14.3 \\
Severe & 28 & 36.4 \\
Extremely severe & 77 & 100.0 \\
Total & & \\
Stress & 21 & 28.3 \\
Normal & 10 & 13.0 \\
Mild & 20 & 26.0 \\
Moderate & 16 & 20.8 \\
Severe & 10 & 13.0 \\
Extremely severe & 77 & 100.0 \\
Total & &
\end{tabular}

\section{Discussion}

This study has demonstrated the negative psychological impact of the COVID-19 pandemic on nurses working in the rural Appalachian region of West Virginia and Kentucky. In this study it was found that $66.3 \%$ of the nurses self-reported their level of depression between a moderate to extremely severe level, $72.8 \%$ of participants reported their anxiety level between moderate to extremely severe, and $58.5 \%$ reported their stress levels between moderate to extremely severe when caring for COVID-19 patients. Compared to healthcare providers and nurses who practiced outside the 
US, the results of this study found that nurses in the rural Appalachian region report having more than twice the level of depression, anxiety, and stress during the COVID-19 pandemic. ${ }^{[6-11]}$ A study conducted in California did have similar results to this study with over $75 \%$ of nurses reporting depression and anxiety. ${ }^{[14]}$ In addition, this study found that nurses with a higher level of education (MSN or higher) reported less depression and anxiety than nurses with lower levels of education. Another study conducted in the US, also found that nursing students, licensed practical nurses (LPN), and clinical assistants had higher levels of depression and anxiety than registered nurses and nurse practitioners. ${ }^{[14]}$

Nurses caring for patients in the Appalachian region have had additional challenges to face besides those mentioned in the research. The supply of primary care physicians in the Appalachian Region is $12 \%$ lower than the national average. ${ }^{[3]}$ By having less physicians, this can limit patients' access to quality healthcare. In addition, many individuals in the Appalachian region live in rural areas where public transportation is unavailable. Both issues can cause patients to delay seeking medical treatment. Also, obesity, smoking, and inactivity are higher in the Appalachian region than in the nation, placing this population at increased risk for heart disease, cancer, chronic obstructive pulmonary disease (COPD), stroke, and diabetes. ${ }^{[3]}$ In addition, nearly $20 \%$ of those in rural counties are aged 65 and older. Older individuals with obesity and health concerns such as heart disease, cancer, diabetes, and COPD are more likely to be seriously ill with COVID-19. ${ }^{[1]}$ Therefore, nurses caring for patients in the Appalachian region have been challenged with limited resources and caring for seriously ill patient with the COVID-19 disease.

During past epidemics, nurses who cared for infectious patients were found to experience greater distress than other healthcare providers due to their direct, intense, and sustained contact with patients. ${ }^{[17]}$ Therefore, it is no surprise that nurses working directly with COVID-19 patients would have higher levels of anxiety and stress considering the lack of information known about the COVID-19 disease. In several studies, the concerns reported by nurses were fear of the unknown, feeling vulnerable, worrying about getting infected, or transmitting the disease to family members or loved ones. $^{[8,9,11,13]}$ In addition, physical signs of stress such as insomnia, dizziness, lack of appetite, and abdominal distress have been reported by nurses when caring for COVID-19 patients during the pandemic. ${ }^{[7,12]}$

A literature review of sixteen studies focused on the impact stress had on nurses working in hospitals related to job satisfaction, job performance, and quality of nursing care.

Published by Sciedu Press
The results of this review found that high levels of stress in nursing staff can result in poor performance, increased turnover, and may adversely affect patient care. ${ }^{[19]}$ This review also found stress increased attrition, reduced retention, and lessened recruitment among nurses. Throughout the US, hospitals continue to report nursing shortages in many departments. Based on the results of this study, hospitals should focus on strategies that will reduce stress in nursing staff to improve retention and job performance.

Healthcare facilities can address the negative impact of the pandemic on nurses by focusing on organizational support, psychological and mental support services, self-care strategies, resilience building and stress management interventions, and promoting social support. ${ }^{[12]}$ Organizational support would focus on clear and timely communication, providing a safe work environment with adequate staffing and PPE, and additional training for caring for COVID-19 patients. ${ }^{[9]}$ Healthcare providers and nurses involved in direct care of COVID-19 patients should have their mental health screened at regular intervals. They should be provided with mental health resource and support when needed. ${ }^{[9,23]}$ Organizations should promote self-care in nurses by offering flexible or shorter work hours, provide adequate breaks and more personal or vacation paid time off (PTO). ${ }^{[12]}$ In one study, the building of resilience in healthcare workers during the COVID-19 was found to preserve their mental and psychological health and well-being. ${ }^{[21]}$ Resilience is the ability to face adverse situations, remain focused, and continue to be optimistic for the future. Resilience can be built through formal education, social support, and meaningful recognition. ${ }^{[22]}$ Finally, social support is essential when facing anxiety-provoking events. Social support can occur between colleagues, friends, and families though phone calls, social media, video conferences, or face-to-face conversations. Nurses' mental health and psychological well-being should be supported through sharing of work experiences, listening to nurses' concerns and offering empathetic support. ${ }^{[12]}$

\section{Limitations}

There were a few limitations identified within the study. The first, was the use of cross-sectional nature of the study which did not allow for interpretation of cause. Additional research would need to be conducted to determine the underlying causes for the high levels of depression, anxiety, and stress reported by the nurses in the study. In addition, there was no baseline mental health history collected on the participants prior to the study being conducted to compare to the results. Therefore, there was no way of knowing if participations had pre-existing mental health issues such as depression, anxiety, or stress before the pandemic. The study was self- 
administered, and symptoms reported by the participants were not verified which may have led to response bias. This study only provided a snapshot of the participants' experience, with no information on the long-term impact of the pandemic on the participants. Furthermore, the study utilized a convenience sampling technique and a small number of participants $(n=77)$ completed the survey making generalization of results different to apply to a larger diverse populations.

\section{Conclusion}

The impact of the COVID-19 pandemic has not been isolated to one region, group, or nationality, but is worldwide prob- lem that continues to affect many lives. To our knowledge, this is the first study to assess the psychological impact of the COVID-19 pandemic on rural Appalachian nurses. In this study, nurses working directly with COVID-19 patients reported high levels of depression, anxiety, and stress. The results of this study were concerning to the authors with the hope for future research to develop strategies and interventions to improve nurses' physical and mental health during times of outbreaks of disease or adverse events.

\section{CONFLiCTS OF InTEREST DisClosure}

The authors declare that there is no conflict of interest.

\section{REFERENCES}

[1] Centers for Disease Control and Prevention. Basics of COVID19 [Internet]. US Department of Health and Human Resources; 2019 [cited 2021, September 3]. Available from: https://www.cdc.gov/coronavirus/2019-ncov/your-hea lth/about-covid-19/basics-covid-19.html

[2] World Health Organization COVID-19 Dashboard [Internet]. WHO Health Emergency Dashboard. [cited 2021, September 3].Available from: https://covid19. who.int/

[3] Appalachian Regional Commission. County economic status in Appalachia, FY 2019. [Internet]. Appalachian Regional Commission. [cited 2021, August 23]. Available from: https ://www .arc.gov/ map/county-economic-status-in-appalachia-fy-2019/

[4] West Virginia Department of Health and Human Resources. (2021, November 14). COVID Disease 2019 (COVID-19). Available from: https://dhhr.wv.gov/COVID-19/Pages/default.aspx

[5] Kentucky Cabinet of Health and Family Services. Kentucky coronavirus monitoring. 2021. https://govstatus . egov. com/kycov id194

[6] Pappa S, Ntella V, Giannakas T, et al. Prevalence of depression, anxiety, and insomnia among healthcare workers during the covid -19 pandemic: A systemic review and meta-analysis. Brain, Behavior, and Immunity. 2020; 8: 901-907. PMid:32437915 https: //doi.org/10.1016/j.bbi.2020.05.026

[7] Arafa A, Mohammed Z, Mahmoud O, et al. Depressed, anxious, and stressed: What have healthcare workers on the frontline in Egypt and Saudi Arbia experience during the COVID 19 pandemic? Journal of Affective Disorders. 2021; 278: 365-371. PMid:33007626 https://doi.org/10.1016/j.jad.2020.09.080

[8] Hummel S, Oetjen N, Du J, et al. Mental health among medical professionals during the COVID-19 pandemic in eight European countries: Cross-sectional survey study. J Med Internet Res. 2021; 23(1): e24983. PMid:33411670 https://doi.org/10.2196/24983

[9] Pandey A, Sharma C, Chapagain R, et al. Stress, anxiety, depression and their associated factors among health care workers during COVID -19 pandemic in Nepal. Journal of Nepal Health Res Council. 2020; 18(4): 655-660. PMid:33510505 https://doi.org/10.3 $3314 /$ jnhrc.v18i4.3190

[10] Tiete J, Guatteri M, Lachaux A, et al. Mental health outcomes in healthcare workers in covid-19 and non-covid-19 care units: a cross sectional survey in Belgium. Frontiers in Psychology. 2021. PMid:33469439 http://doi .org/10.3389/fpsyg. 2020 .612241
[11] Hammond NE, Crowe L, Abbenbroek B, et al. Impact of coronavirus disease 2019 pandemic on critical care healthcare workers' depression, anxiety and stress levels. Australian Critical Care. 2021; 34: 146-154. PMid:33632606 https://doi.org/10.1016/j . au cc. 2020.12 .004

[12] Labrague LJ, De Los Santos J. COVID-19 anxiety among front-line nurses: Predictive role of organizational support, personal resilience and social support. Journal Nurse Management. 2020; 7: 1653-1661. PMid:32770780 https://doi.org/10.1111/jonm.13121

[13] National Nurses Union. National RN survey highlights continued hospital failures to prioritize nurse and patient safety during pandemic. [Internet]. National Nurses Union; 2010-2020. [cited 2021, August 3, 2021]. Available from: https://www national nursesunited.org/press/fifth-survey-of-national-n urses-highlights-continued-hospital-failures

[14] Serrano J, Hassamal S, Hassamal S, et al. Depression and anxiety prevalence in nursing staff during the COVID-19 pandemic. Nursing Management. 2021, Jun 1; 52(6): 24-32.

[15] Arnetz JE, Goetz CM, Arnetz BB, et al. Nurse reports of stressful situations during the COVID-19 pandemic: Qualitative analysis of survey responses. International Journal of Environmental Research and Public Health. 2020; 17(21): 8126. PMid:33153198 https://doi.org/10.3390/ijerph17218126

[16] Lovibond S, Lovibond P. Manual for the depression anxiety stress scale. 2nd ed. Sydney: Psychological Foundation. 1995. https: //doi.org/10.1037/t01004-000

[17] Holton S, Wynter K, Trueman M, et al. Psychological well-being of Australian hospital clinical staff during the COVID-19 pandemic. Australian Health Review. 2020; 45: 297-305. PMid:33032681 https://doi.org/10.1071/AH20203

[18] Coker O, Coker A, Sanni D. Psychometric properties of the 21-item Depression Anxiety Stress Scale (DASS-21). African Research Review. 2018; 12(2): 135-142. https://doi .org/10.4314/afrrev . v12i2.13

[19] Kumar SMY, Bhalla P. Stress among nursing staff in hospitals and its relation with job satisfaction, job performance and quality of nursing care: A literature review. Journal of Nursing and Care. 2019; 8: 492.

[20] Koh D. Occupational risks for COVID-19 infection. Occupational Med. 2020, March; 70(1): 3-5. PMid:32107548 https ://doi .or $\mathrm{g} / 10.1093 /$ occmed/kqaa036

[21] De Brier N, Stroobants S, Vandekerckhove P, et al. Factors affecting mental health of health care workers during coronavirus dis- 
ease outbreaks: A rapid systematic review. Psyarxiv. 2020. https: //doi.org/10.31234/osf.io/w9uxs

[22] Kester K, Wei H. Building nurse resilience. Nursing Management. 2018, June; 49(6): 42-45. PMid:29846293 https ://doi .org/10 $.1097 / 01$. NUMA . 0000533768.28005 .36
[23] Riaz B, Rafai WA, Ahmad U, et al. The psychological impact of COVID-19 on healthcare workers in Pakistan. Future Healthcare Journal. 2021; 8(2): e.293-8. PMid:34286201 https://doi .org/ $10.7861 / \mathrm{fhj} .2020-0193$ 